\title{
QUANTUM OPTICS OF A BOSE-EINSTEIN CONDENSATE
}

\author{
M. LeWenstein*, L. You, J. CoOper \\ Joint Institute for Laboratory Astrophysics \\ University of Colorado and National Institute of Standards and Technology \\ Boulder, Colorado 80309-0440, USA
}

\begin{abstract}
We present a review of recent experimental and theoretical attempis to realize and investigate Bose-Einslein condensation in systems of cooled alkali atoms. We discuss a second quantized theory that describes quantum optics of such systems. We study in detail: (a) the weak field scattering off the condensate; and (b), the probing of the condensate with short laser pulses.
\end{abstract}

PACS numbers: 32.70.Jz, 42.50.Fx, 32.80.-t

\section{Introduction}

Fashion has been a very important motivation of people's activity in general, and in science in particular. One such "scientific fashion" that came and went several times during the last half of the century concerns Bose-Einstein condensation (BEC). The most recent return of the BEC fashion deals with various systems of trapped and cooled atoms [1]. Several experimental groups have attempted to rcalize the BEC [2-4] in such systems. According to experimentalists [5], within onc year we should at least know whether the conditions for condensation are experimentally accessible.

Independent of whether this goal is achieved or not, reliable methods must be developed to detect and diagnose dense systems of very cold atoms. In particular, the quantum statistical character of atoms as bosons or fermions may start to play a crucial role under such conditions. For this reason, we tend to view the theory that we describe below as a new area of quantum optics and atomic physics quantum field theory of atoms interacting with light, or, as some authors call it, nonlinear alom optics [6]. So far the literature on the subject is not very large. Refs. [7-11] deal with the problem of light scattering off a condensate. Lenz et al. describe nonlinear quantum statistical effects in the propagation of atomic waves. Cirac et al. [12] study cooling processes in very small traps that lead to an infinite

*Permanent address: Centrum Fizyki Teoretycznej, Polska Akademia Nauk, Al. Lotników 32/46, 02-668 Warszawa, Poland. 
sequence of condensations. In contrast, note the number of papers accepted by or submitted to Physical Review Letters in this year!

The present paper has the character of review and is organized as follows. First, in Sec. 2, we outline some basic facts about Bose-Einstein condensation. Then, in Sec. 3 we describe various experiments attempting to achieve BEC. In Sec. 4 we focus on experiments. on cold alkali atoms in traps. We present basically "the view from JILA" and describe experiments being done there [13]. In Sec. 5 we introduce the quantum optics of BEC and explain its goals and tasks. Section 6 contains a short discussion of the regimes of parameters accessible experimentally. In Sec. 7 we describe the second quantized theory of a system of cold atoms in a trap. Finally, in Secs. 8 and 9 we discuss two examples of scattering processes off such a system: weak field scattering [11], and scattering of short, but intense laser pulses [10]. We conclude in Sec. 10.

\section{Bose-Einstein condensation}

The aim of this section is to remind the reader of basics of the theory of BEC. We base our discussion on the seminal paper by de Groot et al. [14]. Let us consider a system of non-interacting particles (bosons) of mass $M$ in a box. Assume that the system is in thermal equilibrium with its surroundings. According to general laws of statistical mechanics, the state of the system can then be described by the Bose-Einstein distribution (BED),

$$
N\left(E_{i}\right)=\frac{z \mathrm{e}^{-E_{i} / k_{\mathrm{B}} T}}{1-z \mathrm{e}^{-E_{i} / k_{\mathrm{B}} T}} .
$$

$N_{i}=N\left(E_{i}\right)$ denotes here the mean number of particles occupying the state of energy $E_{i}, k_{\mathrm{B}}$ is the Boltzmann constant, and $T$ is temperature. For simplicily, we assume that the (nondegenerate) ground state energy $E_{0}$ is zero. $z$ denotes the, so called, fugacity, and is related to the chemical potential $\mu \leq 0$ via the relation $z=\exp \left(\mu / k_{\mathrm{B}} T\right)$. There is an additional constraint in the system, due to the fact that the number of particles in the box, $N$, is fixed. Therefore

$$
\sum_{i} N_{i}=N \text {. }
$$

Alternatively we may write the above equation as

$$
\sum_{i} \frac{z \mathrm{e}^{-E_{i} / k_{\mathrm{B}} T}}{1-z \mathrm{e}^{-E_{i} / k_{\mathrm{B}} T}}=N_{0}+\sum_{i \geq 1} N_{i}
$$

The latter equation may be viewed as an equation determining the chemical potential $\mu$ (or fugacity $z$ ) as a function of $T$ and $N$. For example, in a box of linear size $a$ the energies are

$$
E_{i}=\frac{h^{2}}{8 M}\left(\frac{\left(i_{1}+1\right)^{2}+\left(i_{2}+1\right)^{2}+\left(i_{3}+1\right)^{2}-3}{a^{2}}\right),
$$

where $i_{1}, i_{2}, i_{3}=0,1,2, \ldots$ The corresponding plot of fugacity is shown in Fig. 1a. It was Albert Einstein [15] who observed in 1925 interesting properties of this system in the thermodynamic limit as $N$ and $a$ tend to infinity, such that 
the particle density $n=N / a^{3}$ remains constant. As we see from Fig. 1a, there is a critical temperature $T_{0}$ below which $z=1$. In the low temperature phase a macroscopic number $N_{0}$ of particles condense in the ground state (see Fig. 1b). For the case of particles in a box $N_{0} / N$ behaves as $1-\left(T / T_{0}\right)^{3 / 2}$.
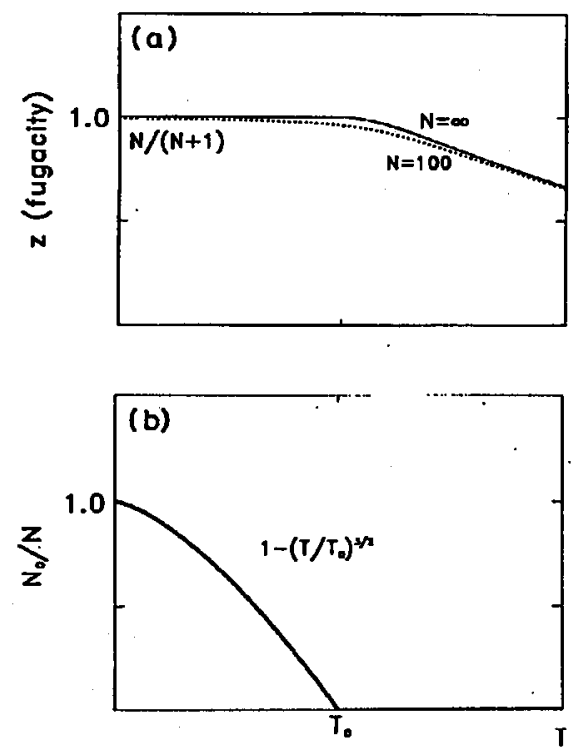

Fig. 1. (a) The temperature dependence of fugacity is shown. Dashed line denotes the result for a finite system with $N=100$, while the solid line represents the result in the thermodynamic limit with same fixed density; part (b) shows the temperature dependence of the condensation.

This phase transition has been termed Bose-Einstein condensation and has created a lot of discussion and interest $[16,17]$. It has rather unusual properties: it occurs in a system of noninteracting particles and is thus a direct consequence of quantum statistics; there is no spatial phase separation, no latent heat, and even no discontinuity in the specific heat. For potentials other than the box potential there are problems with defining the thermodynamic limit since the size of the system is not so precisely defined [14].

There is an heuristic approach to the problem of BEC, which although it is not as precise, it gives a lot of insight into the physics of the problem. Namely, for any potential in the "thermodynamic" limit, we may assume that the energy level spacings are small and substitute sums by integrals in Eq. (3). Introducing the density of states $\rho(E)$ we obtain

$$
N=N_{0}+\int_{0+}^{\infty} \mathrm{d} E N(E) \rho(E),
$$

where $0^{+}$denotes the exclusion of the ground state. 
For instance, for any potential in 3D we have

$$
\rho(E)=\frac{2 \pi(2 M)^{3 / 2}}{h^{3}} \int_{V \cdot(E)} \mathrm{d}^{3} r \sqrt{E-V(r)},
$$

where $V^{*}(E)$ denotes the accessible region in configuration space. For the case of a box in $3 \mathrm{D}$ we thus have

$$
\rho(E)=\frac{2 \pi(2 M)^{3 / 2}}{h^{3}} V \sqrt{E}
$$

where $V$ is the volume of the box. Inserting the above expression to Eq. (5) and letting $\mu \rightarrow 0, T \rightarrow T_{0}$, we find the analytic expression

$$
N=\frac{2.612 V}{\Lambda\left(T_{0}\right)^{3}}
$$

where

$$
\Lambda(T)=\frac{h}{\left(2 \pi M k_{\mathrm{B}} T\right)^{1 / 2}}
$$

is the thermal de Broglie wavelength, i.e. the de Broglie wavelength of a particle that has kinetic energy equal to $k_{\mathrm{B}} T$.

Equation (9) has very important physical consequences, since it is qualitatively valid for any potential [18]. It states that the BEC occurs when the density of atoms is such that their thermal de Broglie wavelengths overlap. This is the parameter region in which one should start looking for condensation. Unfortunately, present experiments on cold atoms are at best two orders of magnitude away from the transition region, either in terms of densities or tempcratures.

\section{Experiments on BEC}

Since the discovery of the BEC, experimentalists have been trying to realize it in the laboratory. The first candidate was liquid helium $\mathrm{He}^{4}$, which undergoes a superfluid transition at low temperatures [19]. Bogolyubov proposed in 1947 [20] to describe this system as a dilute, weakly interacting Bose gas. Bogolyubov's theory did predict some features of the superfluid transition quite well, but soon it was realized that its agreement with experimental data was rather accidental. Liquid helium $\mathrm{He}^{4}$ is in fact characterized by very strong interactions between the atoms, and the phase transition is characterized by the, so called, off-diagonal long range order (ODLRO) [21] and cannot be really identified with BEC (see [19]).

The BEC came to fashion again in 1980, when Hulin et al. [22] discovered that excitons in $\mathrm{Cu}_{2} \mathrm{O}$ obey Bose-Einstein statistics of a (practically) ideal gas. There are two kinds of such excitons, ortho-excitons with spin 1, and para-excitons with spin 0 . Both kinds are characterized by high mobility and relatively long life times. Since 1980 many experiments have been done, but only recently, has the $\mathrm{BEC}$ of para-excitons in stressed $\mathrm{Cu}_{2} \mathrm{O}$ apparently been observed [23].

Another candidate for BEC is a gas of spin polarized hydrogen atoms, studied by the groups at MIT, Harvard and in Amsterdam [3, 4, 24]. In this case one can try a straightforward method - put the gas into a box with the piston, cool it and compress it in order to achieve the required density. Unfortunately, hydrogen 
atoms have a tendency to stick to walls and form solid hydrogen clusters. These problems might be overcome by using non-stick walls. Despite valiant attempts, however, the BEC of spin polarized hydrogen has not yet been observed.

Finally, several groups $[2,13,25]$ are attempting to realize the $\mathrm{BEC}$ in a system of cooled alkali atoms using combinations of recently developed methods. A toms are trapped optically and cooled using the Doppler cooling to about $250 \mu \mathrm{K}$. Atomic molasses are then formed and cooled further with the help of the polarization gradient cooling and the Sisyphus effect. This technique allows in principle velocities of the order of $v \approx 3 \hbar k_{L} / M$ to be achieved, where $k_{L}$ is the photon wave number. This corresponds roughly to the energy of photon recoil and a temperature of the order of few $\mu \mathrm{K}$. All of these methods are thoroughly discussed in Ref. [1]. Also, magneto-optical traps (MOT's), such as the ones at JILA [13], combined with the method of evaporative cooling are being used. We discuss these techniques in detail below.

\section{Cooled atoms in traps}

Magnetic traps are based on a very simple principle. Energy levels of alkali atoms in magnetic field undergo Zeeman splitting. The potential energy in the magnetic field $\boldsymbol{B}$ is just $-\boldsymbol{\mu} \cdot \boldsymbol{B}$, where $\boldsymbol{\mu}$ is the total magnetic moment. A typical behavior of energy levels is represented in Fig. 2. There are "weak field seekers", that can be confined to local minima of $B$, and "strong field seekers", which actually cannot be trapped in static fields.

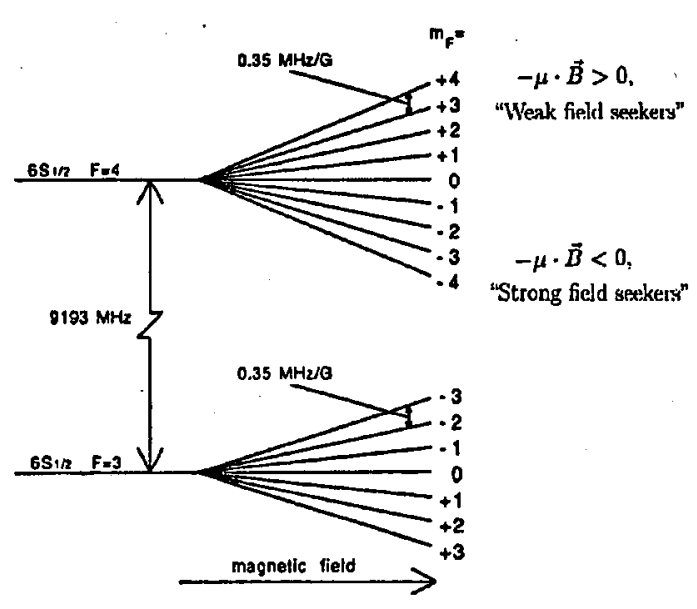

Fig. 2. Typical level structures of the trapping states, illustrated by cesium $6 S_{1 / 2}$ Zeeman energy level structure.

In the experiments, the magnetic field is appropriately designed to form an effective potential, which can be well approximated by a harmonic potential close to its minimum. The potential forms, however, a finite barrier. Both the 
height of the barrier, as well as the curvature of the potential at the minimum, which determines the frequency of atomic motion in the trap, can be controlled. The experimental set up used in JILA is shown schematically in Fig. 3. Note the anti-IIelmholtz coils that counteract the gravitational force.

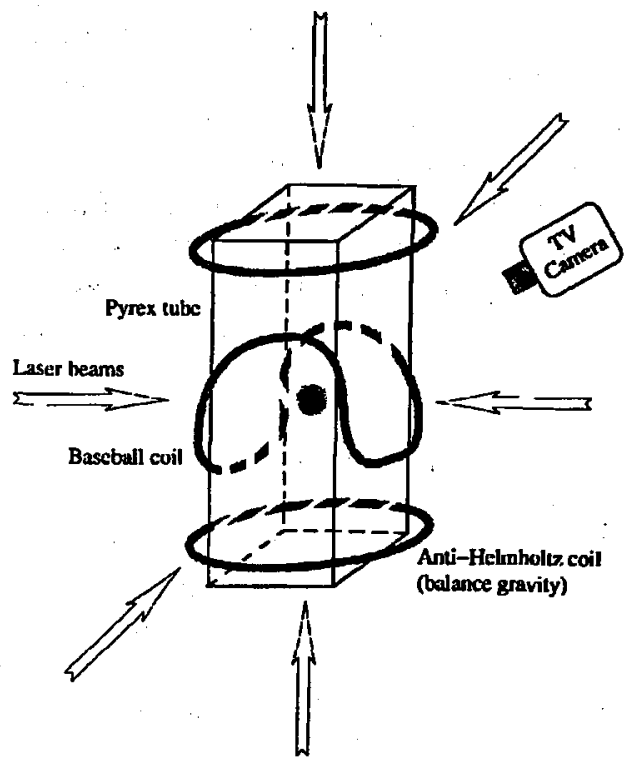

Fig. 3. Illustration of a baseball coil magnetic trap configuration. The baseball coil has a radius of $2.25 \mathrm{~cm}$. The pair of anti-Helmholtz coils generates a magnetic field gradient which cancels gravity.

The fact that the potential has a finite barrier is used for the evaporative cooling, first developed by Kleppner's group at MIT. Suppose we have some atoms in the trap at the temperature $T$. When one lowers the barrier to the height of few $k_{\mathrm{B}} T$, the hottest atoms leave the trap, and cooling occurs. Although one loses part of the atomic population, the system is cooled very effectively to a few $\mu \mathrm{K}$ or even less. Experimentalists hope that the use of MOT's and evaporative cooling will allow for realization of the BEC of cooled alkali atoms.

\section{Quantum optics of a Bose-Einstein condensate}

We now turn to the main topic of this review, i.e. the quantum optics of a BEC. We shall not discuss any more, how to achieve and realize the condensation. We shall assume that it is possible and try to answer the question of how to probe and diagnose the system in the condensed state. Obviously, this task should be achievable using optical methods, such as light scattering off the system. In order to describe such processes, however, we have to develop an appropriate theory that takes into account the quantum statistical properties of atoms as bosons. A natural framework for such a theory is provided by second quantization and other 
standard methods of many body physics [19]. From that point of view, such a theory has a more general scope - it describes not only quantum optics of BEC, but is, generally speaking, a quantum field theory of cooled atoms interacting with light.

After introducing the second quantized theory, we will discuss two examples of its applications: weak field scattering off the BEC, and scattering of short intense pulses off the system of cooled atoms.

\section{Regimes of parameters}

Before we start the discussion of the quantum field theory of the atoms, it is useful to specify regimes of parameters that are accessible experimentally, in particular for the magneto-optical trap developed at JILA $[2,13]$, discussed above. The potential for the atomic center-of-mass motion for a single atom in its ground clectronic state can be well described by a harmonic oscillator potential of frequency $\omega_{t} \approx(2 \pi) 10 \mathrm{IIz}$. Although the potential forms a finite barrier only, several thousand energy levels exist within the trap. By exploiting the evaporative cooling technique, the trap can store about $10^{8}$ cesium atoms, which will interact with resonant light of frequency $\sim(2 \pi) 4.0 \times 10^{14} \mathrm{~Hz}$. A typical photon recoil energy will then be $\approx(2 \pi) 2 \mathrm{kIIz}$, whereas the natural linewidth (IIWIIM) $\gamma \approx(2 \pi) 2.5 \mathrm{MIIz}$. The size of the ground statc wave function for the condensate is expected to be $a \geq 10^{-5} \mathrm{~m}$, in comparison with the resonant wavelength, $\lambda \approx 800 \mathrm{~mm}$. We note that $a$ for the ground state of the condensate is several times bigger than $1 / \sqrt{2 M \omega_{t}}$ with $M$ being the mass of Cs. The $a$ we use takes into account the (expccted) repulsive ground state interactions, which are believed to increase the size of the condensate [26]. Such a change in potential is equivalent to replacing $\omega_{l}$ by an effective trap frequency $\omega_{\text {eff. }}$. Both $\omega_{\text {eff }}$ and $a=1 / \sqrt{2 M \omega_{\text {eff }}}$, can to some extent be controlled in the experiment. In general, atoms in excited electronic states move in different potentials from that characterizing the ground state. In this paper, we consider the case of no potential in the excited state, and comment on other cases.

\section{Second quantized theory}

The quantum field theory of atoms interacting with light can be constructed using standard tools of many body physics. We start from first quantized thcory, and then introduce appropriate sets of atomic creation and annihilation operators etc. The details of the construction are described in Ref. [27].

The IIamiltonian governing the evolution of atoms in the trap takes the following second quantized form in the rotating wave approximation (RWA) and in atomic units:

$$
\begin{aligned}
\mathcal{H} & =\sum_{n} E_{n}^{g} g_{n}^{\dagger} g_{n}+\sum_{m}\left(E_{m}^{e}+\omega_{0}\right) e_{m}^{\dagger} e_{m}+\sum_{\mu} \int \mathrm{d}^{3} k c k a_{k_{\mu}}^{\dagger} a_{k \mu} \\
& +\sum_{n, m} \sum_{\mu} \int \mathrm{d}^{3} k \varrho(k)\left[\eta_{n m}(k) g_{n}^{\dagger} a_{k_{\mu}}^{\dagger} e_{m} \cdot \varepsilon_{k_{\mu}}+\text { h.c. }\right]+\mathcal{H}_{c}
\end{aligned}
$$


where $g_{n}, g_{n}^{\dagger}$ denote atomic annihilation and creation operators for the $n$-th state of the ground state potential. For a rotationally invariant potential, $n$ is actually a triple index $\left(n_{x}, n_{y}, n_{z}\right)$. The corresponding energy is $E_{n}^{g}=\omega_{t}\left(n_{x}+n_{y}+n_{z}\right)$. $\boldsymbol{e}_{\boldsymbol{m}}, \boldsymbol{e}_{\boldsymbol{m}}^{\dagger}$ denote atomic annihilation and creation operators in the excited state potential. In the case of no potential they annihilate and create plane waves of momentum $m$. The corresponding energies are $E_{m}^{e}+\omega_{0}=m^{2} / 2 M+\omega_{0}$, i.e., are shifted by the electronic transition frequency. We consider here the case of a transition from an $s$-state to a $p$-state and therefore the $e_{m}$ 's and $e_{m}^{\dagger}$ 's have a corresponding $s-p$ vector character. This is not the case for the transition in a typical alkali atom, but the character of the transition is not essential for our conclusions. $a_{k_{\mu}}$ and $a_{k \mu}^{\dagger}$ denote annihilation and creation operators for photons of momentum $\boldsymbol{k}$ and linear polarization $\varepsilon_{\boldsymbol{k}_{\mu}}(\mu=1,2)$. All operators fulfill standard bosonic commutation relations. The coupling $\varrho(k)$ is a slowly varying function of $k$ related to the natural linewidth $\gamma=\left(8 \pi^{2} k_{0}^{2} / 3 c\right)\left|\varrho\left(k_{0}\right)\right|^{2}$, with $k_{0}=\omega_{0} / c$. The matrix elements $\eta_{n m}(k)$ describe the transition from the $n$-th state of the ground state potential to the $m$-th state of the excited state potential,

$$
\eta_{n m}(k)=\left\langle n\left|\mathrm{e}^{-\mathrm{i} k \cdot R}\right| m\right\rangle \text {. }
$$

These matrix elements are analogous to the Franck-Condon factors.

The last part of the IIamiltonian, which has to be included when the dipole approxirnation is used [28] is usually neglected, since it describes the contact interaction between atoms in the excited and ground states. In the case of atoms moving or even condensing inside a trap, such a procedure cannot be justified, since the atomic wave functions may well overlap. Only if the contact term is taken into account, does the total Hamiltonian include fully the strong resonant atomic interactions due to electronic dipole-dipole forces and exchange of transverse photons* [29].

In the first quantization picture the contact term has the form

$$
\mathcal{H}_{\mathrm{c}}^{I}=4 \pi e^{2} \sum_{i \neq j} x_{i} \cdot \boldsymbol{x}_{j} \delta\left(\boldsymbol{R}_{i}-\boldsymbol{R}_{j}\right)
$$

where $x_{i}$ 's and $R_{i}$ 's are electronic and atomic position operators respectively. In the second quantized form the contact term can be conveniently written in the coordinate representation, when we introduce atomic fields

$$
\begin{aligned}
\psi_{g}(\boldsymbol{R}) & =\sum_{n}\langle\boldsymbol{R} \mid n\rangle g_{n}, \\
\psi_{e}(\boldsymbol{R}) & =\sum_{m}^{n}\langle\boldsymbol{R} \mid m\rangle e_{m},
\end{aligned}
$$

and their Hermitian conjugates. In terms of these fields the contact term is

$$
\mathcal{H}_{\mathrm{c}}=4 \pi d^{2} \int \mathrm{d}^{3} R \psi_{g}^{\dagger}(R) \psi_{e}^{\dagger}(R) \cdot \psi_{e}(R) \psi_{g}(R)
$$

where $d$ is the absolute value of the dipole transition moment.

*The elimination of the electromagnetic field from the theory described by Eq. (10) leads to an effective field theory of interacting atoms, called in Ref. [6] nonlinear atom optics. 
Beyond the dipole approximation, there are other interaction terms that should be added to the Hamiltonian (10) that describe short range collisions of atoms (in ground-ground and excited-excited electronic states). We shall neglect them in the discussion below, although we are fully aware how important they could be, especially since the condensation can only be achieved for a positive scattering length. Perhaps the most challenging task of this new theory is to study the role of inter-atomic interactions in the formation of the condensate and in optics of the system.

\section{Weak light scattering at $T=0$}

The first application of the above theory concerns the scattering of weak, monochromatic laser light off a condensate at zero temperature (see Fig. 4). In such a case all atoms are condensed in the ground state of the center-of-mass motion, and we may expect that the interaction with the laser will only slightly perturb this state.

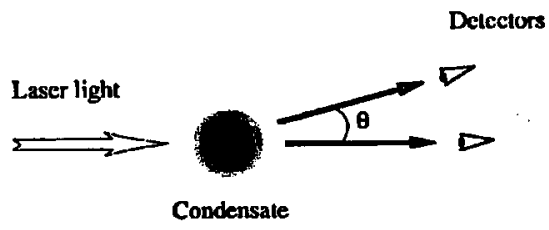

Fig. 4. Schematic illustration of light scattering off a condensate.

Therefore, for this weak light scattering study at $T=0$, we assume a linearization of atomic amplitudes around the ground state, and substitute the $g_{n}$ 's by their appropriate initial mean values $[7,9]$ :

$$
\left\langle g_{n}\right\rangle \rightarrow \delta_{n 0} \sqrt{N} .
$$

This linearization has been used by several authors, and has led to several interesting predictions. Politzer ([7], see also [8]) considered the limit of an infinite trap, i.e. in the absence of any trapping potential. The model then becomes invariant with respect to space translations and momentum must be conserved. In such a case, atomic and photonic degrees of freedom mix, giving rise to a band gap in the excitation spectrum. This is an optical analogue of polariton formation or the Anderson-Iliggs phenomenon [19]. The two branches of the dispersion relation, that occur in some regions of momentum are identified as "photonic" and "atomic", and are shown in Fig. 5. Because of the band gap in the spectrum the resonant light will be strongly reflected back from the sharp boundary of the condensate. Unfortunately, this interesting conclusion does not have much relevance for the traps currently being investigated. As we have noted, they have a finite size (a few microns) and no sharp boundaries. As we shall see below, the light from realistic traps is scattered primarily in the forward direction and only deflected from the forward direction by a small angle of the order of $1 /\left(k_{L} a\right)^{2}$. 


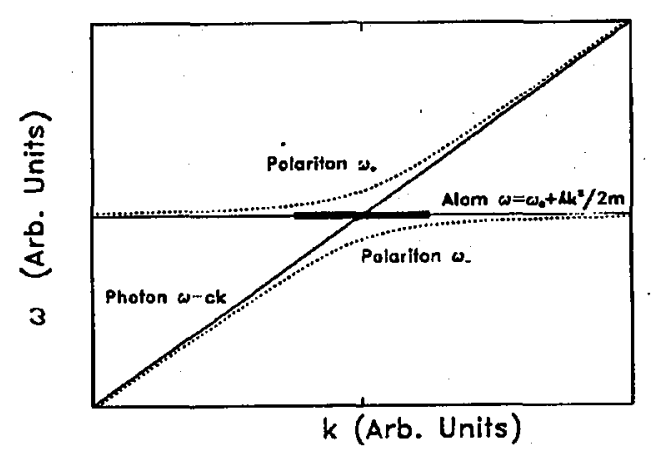

Fig. 5. In the case of no trapping potentials, the photonic degree of freedom (slanted solid line) mixes with the atomic excitation (solid line) to generate two branches of polaritons (represented in dashed lines) due to field-matter coupling. A band gap in frequency (energy) is formed that covers all the $k$ space. Within this gap region, no propagating light modes are supported, thus resulting in a total reflection of incident light.

Javanainen [9] has tried to describe a realistic trap, but made another simplification, namely describing the atomic field as just one harmonic oscillator, corresponding to a collective excitation. In this way he neglected the difference in propagation of different spatial modes through the medium. As a result, in the steady state, a small number of atoms remain in the excited state, and the number of scattered photons has a resonant Lorentzian shape as a function of the frequency of the incident light. The width of this Lorentzian is $\gamma_{\mathrm{eff}}=3 \mathrm{N \gamma} / 2\left(k_{L} a\right)^{2}$, i.e. it is roughly a cooperative spontaneous emission width decreased by the factor $1 /\left(k_{L} a\right)^{2}$, which accounts for the fact that cooperative emission only takes place into a limited cone in the forward direction due to approximate momentum conservation. As we shall see below, Javanainen's theory, in addition to its appealing simplicity, predicts accurately the overall scale of the spectrum, and in fact should work well in the limit of optically thin condensates (i.e. when $a \rightarrow 0$ ).

We have tried to solve this problem [11] without any other approximation apart from the linearization (16). Upon eliminating the atomic excited state operators, a scattering equation for the field amplitude can be derived. It takes the form

$$
\dot{a}_{k_{\mu} \mu}=-\mathrm{i} c k a_{k_{\mu}}-\sum_{\mu^{\prime}} \int \mathrm{d} k^{\prime} \int_{0}^{t} \mathrm{~d} t^{\prime} \mathcal{K}\left(t-t^{\prime} ; k, \mu, k^{\prime}, \mu^{\prime}\right) a_{k^{\prime} \mu^{\prime}}\left(t^{\prime}\right) .
$$

The kernel $\mathcal{K}\left(t-t^{\prime} ; k, \mu, k^{\prime}, \mu^{\prime}\right)$ can be evaluated analytically in the case of no potential or a harmonic potential in the excited state (for details see Refs. [11, 27]). Here we stress only that the kernel in Eq. (17) has a simple physical meaning. It describes the amplitude of the process of absorption of a photon with momentum $k^{\prime}$ at time $t^{\prime}$, accompanied by the formation of a wave packet in the excited state potential. This wave packet then undergoes evolution in the excited state manifold until it recombines to the ground state at time $t$, emitting a photon of the momen- 
tum $k$. The evolution includes the free part and the effect of the contact potential. Note, the free evolution of the wave packet in the time interval $\tau=t-t^{\prime}$ consists primarily in quantum diffusion plus drift due to the momentum of the absorbed photon*. When the wave packet drifts away from the center of the trap and diffuses sufficiently strongly, the recombination accompanied by emission becomes impossible. The kernel $\mathcal{K}\left(t-t^{\prime} ; k, \mu, k^{\prime}, \mu^{\prime}\right)$ thus decays on a characteristic time scale $1 / \Gamma$. It is easy to check that $\Gamma$ must be of the order of $k a \omega_{\text {eff }}=\sqrt{\omega_{\text {eff }} k^{2} / 2 M}$, i.e. the reciprocal of the time to drift a distance $a$.

One might try to solve Eq. (17) using the Born approximation, i.e. treating perturbatively the self-energy term on the right hand side. Such an attempt fails, due to the optical thickness of the condensate. Self-energy terms are extremely important close to resonance and one has to fully account for them. We have solved Eq. (17) numerically in the on-shell approximation to the scattering matrix [30]. We discretize the solid angle and solved the resulting finite set of linear equations. However, we can also construct an approximate analytic solution of Eq. (17) in terms of partial waves, provided we neglect the dependence of the kernel $\mathcal{K}$ on the polarizations $\varepsilon_{k_{\mu}}$. This is a good approximation, since the scattering occurs mainly in the forward direction and the scattered photons have polarizations that are approximately perpendicular to $k_{L}$, and do not couple to one another.

The quantity of interest is the normalized scattering cross-section, i.e. the total number of scattered photons of the frequency $\omega_{L}$, per time and normalized to the total number of photons incident onto the area $\pi a^{2}$ (normalized cross-section). We denoted it by $\sigma$. It is plotted in Fig. 6 for the case of $N=10^{7}$ atoms. The overall scale of the spectrum is the same as predicted by Javanainen, and for the parameters used is of the order of $10 \mathrm{GHz}$. The spectrum, however, is evidently non-Lorentzian and exhibits a very narrow structure at the resonance of the width of the order of $\Gamma \approx 800 \mathrm{~Hz}$.

The narrowing of the spectrum is somewhat reminiscent to Dicke's coherent narrowing [31]. The spectrum is in fact a sum of Lorentzians corresponding to different partial waves. The waves characterized by low angular momenta $l$ contribute with a more or less constant width $\approx \gamma_{\text {eff }}$ to the cross-section. The waves with high $l$ require more and more time to become scattered and contribute with the decreasing widths.

Summarizing, the line shape from the BEC has thus two appealing properties. First, it exhibits a very broad resonance of the width $\gamma_{\text {eff. }}$ In order to detect even a partial condensation it will be sufficient to shine strongly detuned light onto the system of cooled atoms. Those of them which are not in the condensate phase, will be essentially transparent to such a light and will not contribute any undesired background. Condensed atoms will, however, still produce quite a strong signal.

Second, the narrow feature $(800 \mathrm{~Hz})$ in the spectrum at $\omega_{L} \approx \omega_{0}$ suggests obvious applications of this system to precision spectroscopy. This is a rare example of a situation where such narrow resonances are present in the line shape, and yet the response of the system at these resonances is strong, and contrasting strongly

*That is evidently true in the case of no potential in the excited state, but is also true in the case of harmonic potential if $\tau \ll 1 / \omega_{t}$. In the latter case, the wave packet does not have time to reverse its motion and give rise to back scattering. 

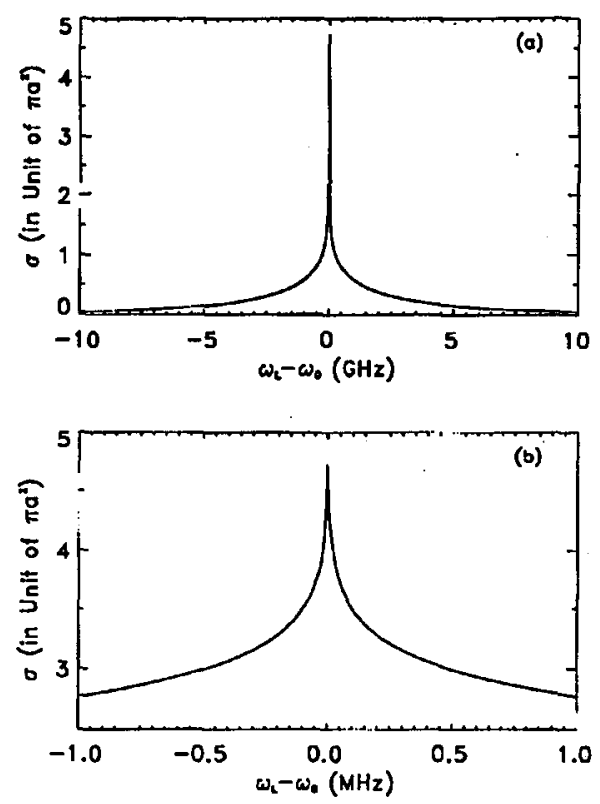

Fig. 6. Scattering cross-section of the BEC for $N=10^{7}$ (other parameters as given in the text). Part (a) shows the overall shape, part (b) is an enlargement of the central region.

with the response far from them. One should stress, however, that our theory neglects several broadening mechanisms (such as for example spontaneous emission to non-condensate states) which may affect the width of the narrow feature.

\section{Scattering of short laser pulses}

The second application of the theory formulated in Sec. 7 concerns scattering of short laser pulses off the system of cooled atoms [10]. If such a pulse is strong enough and short enough, we may neglect both spontaneous emission effects and dipole-dipole interaction, and substitute the electric field operator entering the interaction IIamiltonian in Eq. (10) by a $c$-number. The pulses should have duration $300-10$ ps or shorter, i.e. width $\gamma_{L} \approx 3 \times 10^{9}-10^{11} \mathrm{~Hz}$. The first estimate shows that indeed $\gamma_{L} \gg \gamma$, i.e. the spontaneous emission may be legitimately neglected during the time of interaction of the pulse with the atoms. Note, however, that this estimate might be misleading actually, since the atoms will respond collectively and we should compare $\gamma_{L}$ to the effective spontaneous emission rate $\gamma_{\text {eff. Fortu- }}$ nately, as discussed in previous section, the latter is of the order of few $\mathrm{GHz}$, so that the condition $\gamma_{\mathrm{L}} \geq \gamma_{\mathrm{eff}}$ still holds. We will nevertheless check our assumption self-consistently in the following, by assuring that the total number of emitted photons $N_{\text {tot }}$ is much smaller than $N$ (in this way the probability of spontaneous emission of a given atom is small).

Under these assumptions, we substitute the electric field operator multiplied 
by the absolute value of the electronic transition dipole moment by

$$
d \overrightarrow{\mathcal{E}}^{(+)} \rightarrow \frac{\Omega}{2} \sum_{\mu} \int \mathrm{d}^{3} k \varrho(k, \mu) \mathrm{e}^{\mathrm{i} k \cdot \boldsymbol{R}-\mathrm{i} c k t},
$$

where $\Omega$ is the peak Rabi frequency of the laser pulse. The function $\varrho(k, \mu)$ describes the $(k, \mu)$-dependent envelope of the pulse. We assume that the pulse has the form of a plane wave packet moving in the $k_{L}$ direction with central frequency $\omega_{L}$ and linear polarization $\varepsilon_{L}$, so that

$$
d \overrightarrow{\mathcal{E}}^{(+)} \rightarrow \frac{\Omega}{2} \varepsilon_{L} \mathcal{F}\left(\gamma_{L}\left(t-k_{L} \cdot \boldsymbol{R} / \omega_{L}\right)\right) \mathrm{e}^{\mathrm{i} \boldsymbol{k}_{L} \cdot \boldsymbol{R}-\mathrm{i} \omega_{L} t}
$$

Here, $\mathcal{F}\left(\gamma_{L} t\right)$ is the temporal envelope of the pulse chosen to be real and assumed to have a bell-shape with a maximum at $t=0$ equal to 1 .

The theory simplifies even further when we observe that the ground state annihilation operators couple in the Hamiltonian (10) to the linear combinations of excited state operators

$$
g_{n} \rightarrow \sum_{m} \eta_{n m}(k) e_{m}
$$

For the parameters considered, the momentum spread in the pulse is much smaller than the characteristic scale of momentum change in the Franck-Condon factors $\eta_{n m}(k)$. We may thus substitute $\sum_{m} \eta_{n m}(k) e_{m}$ by $\sum_{m} \eta_{n m}\left(k_{L}\right) e_{m}$ and introduce wave-packet operators

$$
f_{n}=\sum_{m} \eta_{n m}\left(k_{L}\right) e_{m}
$$

and their Hermitian conjugates $f_{n}^{\dagger}$, respectively.

The Heisenberg equations of motion are now linear and at resonance and in the rotating frame take a vcry simple form

$$
\begin{aligned}
& \dot{g}_{n}=-\mathrm{i} \frac{\Omega}{2} \mathcal{F}\left(\gamma_{L} t\right) \varepsilon_{L} \cdot f_{n}, \\
& \varepsilon_{L} \cdot \dot{f}_{n}=-\mathrm{i} \frac{\Omega}{2} \mathcal{F}\left(\gamma_{L} t\right) g n,
\end{aligned}
$$

with other components of $f_{n}$ which are left intact. These equations may be easily solved analytically for any pulse envelope with the initial condition for the ground state populations corresponding to the Bose-Einstein distribution.

The physical picture of the process is the following: each of the $n$-th levels of the ground state oscillator (when populated) create an independent wave-packet $f_{n}$. The population then oscillates coherently between the ground state and $n$-th wave packet. The system behaves as a set of independent two-level atoms coherently driven by the laser pulse. If the area of the pulse is a multiple of $2 \pi$ the system will be left in the same state after the pulse is over as it was before it came. Obviously, as $n$ grows the approximations that we have made become worse, but they should hold very well for the lowest $\sim 10^{4}$ states of the ground state potential that are available.

Of course, in reality the atoms will scatter photons since $\gamma$ is non-zero. The resonance fluorescence (RF) from a single atom driven by a short pulse has been 
studied by Rzażewski and Florjaniczyk [32]. They have shown that the RF spectrum consists of $2 K-1$ peaks, provided the pulse area is $2 \pi K$. Physically multiple splitting results from temporal interference effects, as photons emitted during the interaction with the pulse interfere one with another. These results were then generalized to include non-zero detunings, dissipation and various pulse shapes (hyperbolic secant, exponential pulses, chirped pulses etc.) [33]. The total number of photons emitted in such a process is typically of the order of $\gamma / \gamma_{L}$.

In the present case, the quantities of interest are: the photon spectrum defined as the total number of scattered photons of momentum $k$ and polarization $\mu$ :

$$
C(k, \mu)=\lim _{t \rightarrow \infty}\left\langle a_{k_{\mu}}^{\dagger}(t) a_{k \mu \mu}(t)\right\rangle,
$$

and the total number of scattered photons $N_{\text {tot }}=\sum_{\mu} \int \mathrm{d}^{3} k C(k, \mu)$. The spectrum $C(k, \mu)$ consists of coherent and incoherent parts. In the low temperature limit the coherent part gives the dominant $\left(\propto N^{2}\right)$ contribution to the fluorescence.

For $T>T_{0}$ the coherent part of the spectrum becomes

$$
C_{\mathrm{coh}}(k, \mu)=S_{\mathrm{coh}}\left(\left(c k-\omega_{L}\right) / \gamma_{L}\right) N^{2} \exp \left(-2 a^{2}\left(k-k_{L}\right)^{2} / \beta \omega_{t}\right),
$$

where $S_{\text {coh }}\left(\left(c k-\omega_{L}\right) / \gamma_{L}\right)$ denotes the single atom coherent spectrum [32, 33]. For $T \approx 10 \mu \mathrm{K}, \beta \omega_{t} \approx 5 \times 10^{-5}$ and the scattering will occur practically in the forward direction and will cover only a solid angle with half-angle $\leq 1.0 \times 10^{-4}$. The total number of photons remains very small in this regime. Physically, the reason is that in the high temperature limit the atoms behave as independent random scatterers. The radiation from different atoms interferes destructively in all directions, except in the vicinity of the forward direction where the constructive phase matching takes place. The picture remains essentially the same as $T$ approaches $T_{0}$.

The situation dramatically changes when $T<T_{0}$. The spectrum will contain a new term arising from the condensate. Assuming that on the average $N_{0}$ atoms form a coherent wave packet of width $a$ occupying the lowest energy state with $n=(0,0,0)$, we obtain for the coherent part

$$
C_{\mathrm{BEC}}(k, \mu)=S_{\mathrm{coh}}(\varpi) N_{0}^{2} \exp \left(-a^{2}\left(k-k_{L}\right)^{2}\right) .
$$

As we would expect, the coherent scattering now covers a much larger solid angle with half-angle $\sim 1.0 \times 10^{-2}$. At a distance of $1 \mathrm{~m}$ from the trap the scattered photons will be about $1 \mathrm{~cm}$ from the optical axis. The total number of such photons grows also dramatically as $N_{0}$ grows and $T$ decreases, but remains smaller than $N$ in the considered regime of parameters.

The above results are illustrated in Figs. 7 and 8 . In Fig. 7 we show the dependence of $N_{\text {tot }}$ on $N_{0}$ for 100 ps and 10 ps pulses. Both plots were calculated for a hyperbolic secant pulse of area $2 \pi$ and $N=10^{8}$. Since $N_{0}$ is a unique function of temperature, one can view the plots as representing temperature dependence. The transition point is clearly visible. For $100 \mathrm{ps}$ pulse (and even more so for $10 \mathrm{ps}$ pulse), our theory is valid down to $T=0$, since $N_{\text {tot }} / N$ remains smaller than unity. In Figs. 9 and 10 we show a 3D plot of the scattering amplitude $F(\omega, \theta) / N$ as a function of frequency and scattering angle. The spectrum 


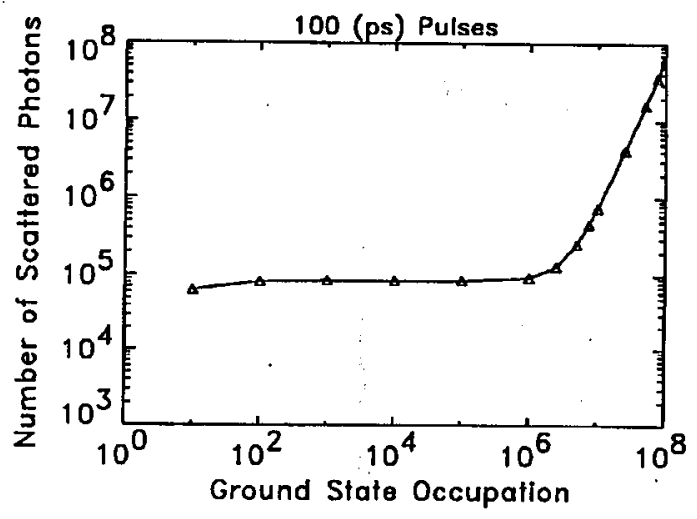

Fig. 7. Dependence of the total number of scattered photons on the condensate occupation for $\tau_{p}=100$ ps.

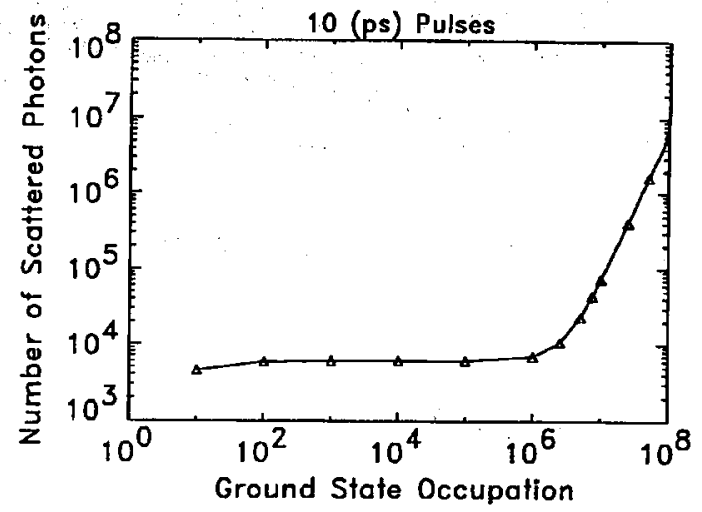

Fig. 8. Dependence of the total number of scattered photons on the condensate occupation for $\tau_{p}=10 \mathrm{ps}$.

is equal to the square of $F(\omega, 0)$, multiplied by the single atom spectrum, i.e. $C_{\text {coh }}(\omega, \theta)=|F(\omega, \theta)|^{2} S_{\text {coh }}(\omega)$. The plot in Fig. 9, corresponds to $N_{0}=10^{-5} N$, i.e. a temperature close to the critical point, whereas in Fig. 10 for $N_{0}=0.5 \mathrm{~N}$ we have $T<T_{0}$. Clearly, the angular distribution changes dramatically and exhibits a broad component that indicates scattering from the condensate.

To summarize this section, we have demonstrated that by scattering from short laser pulses of area $2 \pi K$ we may detect the onset of the Bose-Einstein condensation. In the regime of validity of our theory, $2 \pi K$ pulses leave the system of trapped atoms to a large extent unperturbed. This is particularly true for $T \geq T_{0}$. As $T$ becomes smaller than $T_{0}$, the angular distribution of scattered photons, as well as their number, change dramatically. Scattering of short laser pulses on systems of trapped atoms thus provides an alternative way of detecting the actual state of the system, i.e. its temperature, degree of condensation etc. 
188

M. Lewenslein, L. You, J. Cooper

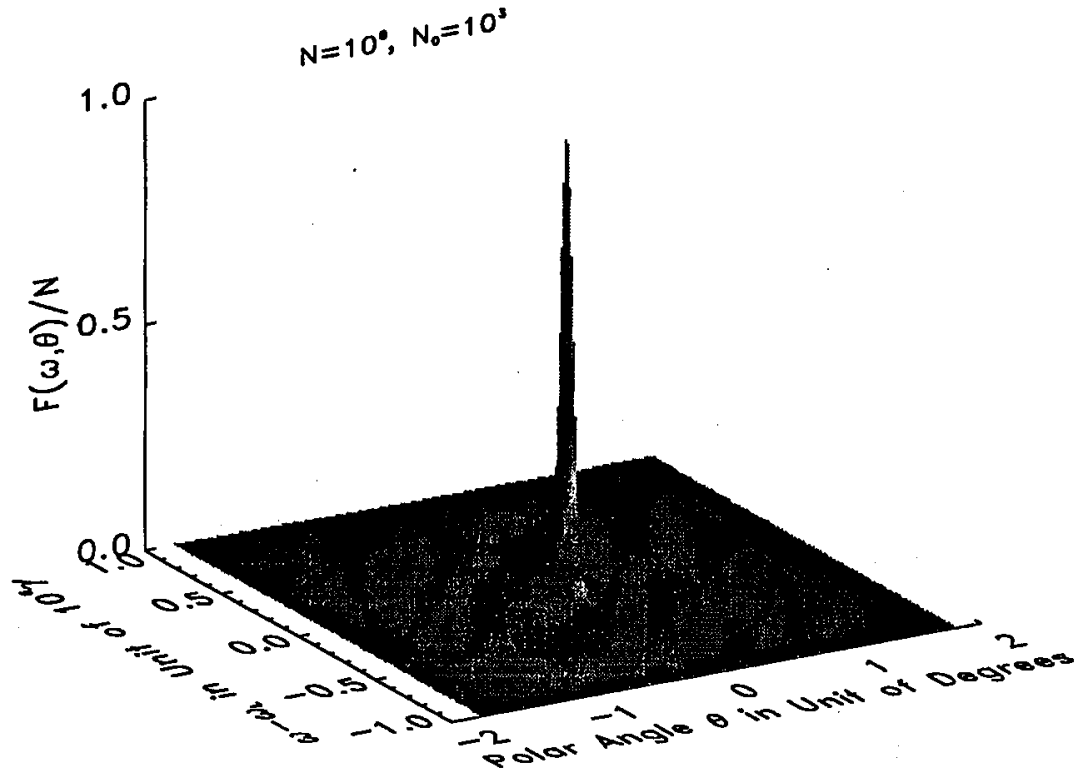

Fig. 9. Scattering amplitude $F(\theta, \omega)$ for $N_{0}=100=10^{-5} \mathrm{~N}$.

$$
N=10^{\circ}, N_{0}=N / 2
$$

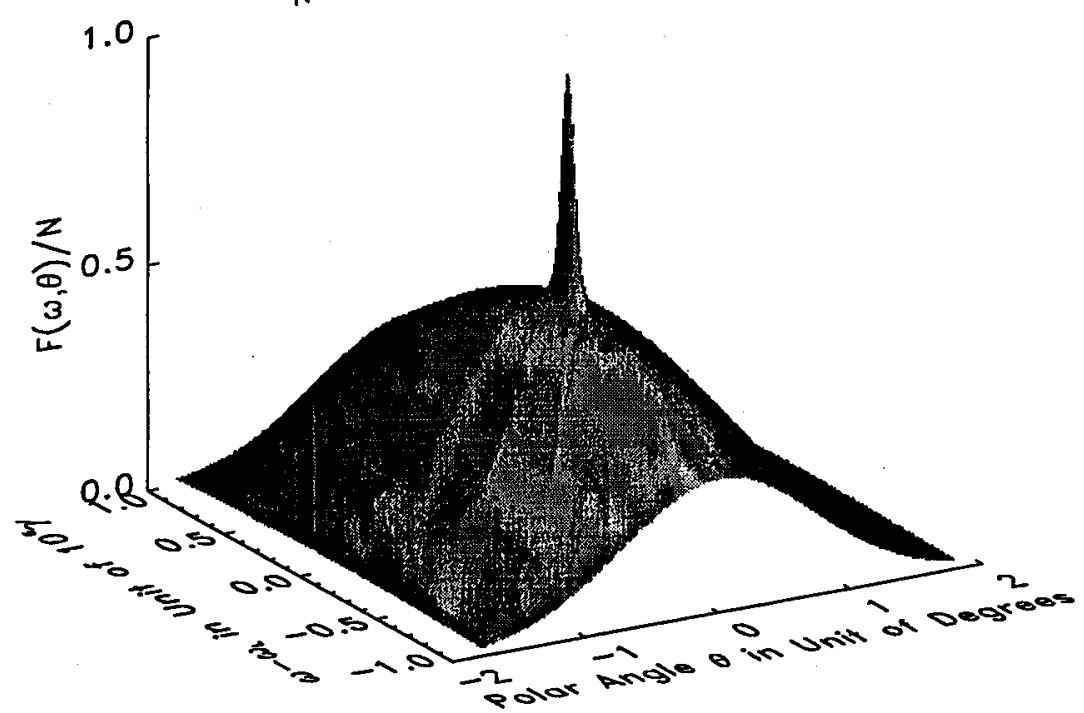

Fig. 10. Scattering amplitude $F(\theta, \omega)$ for $N_{0}=5 \times 10^{7}=N / 2$. 


\section{Conclusions}

We have presented some recent developments of a new area of quantum optics and atomic physics: quantum field theory of atoms interacting with light. The methods of many body physics liave to be introduced and used in this area. Let us, however, also express the hope that, in the future, the applications of tools of quantum optics (such as, for instance, those associated with quantum noise, quantum stocliastic processes and master equations [34]) will contribute to the development of many body physics!

We thank all members of the BEC Seminar at JILA for enlightening discussions and comments. M.L. thanks JILA for hospitality and the financial support of his visiting fellowship. L.Y. and J.C. are supported in part by NSF grant PIIY90-12244 through the University of Colorado.

\section{References}

[1] Eds. S. Chu, C. Wieman, special issue of J. Opt. Soc. Am. B 6, (11) (1989).

[2] C. Monroe, W. Swann, II. Robinson, C. Wieman, Phys. Rev. Lett. 65, 1571 (1990).

[3] H.F. Hess, G.P. Kochanski, J.M. Doyle, N. Masuhara, D. Kleppner, T.J. Greytak, Phys. Rev. Lett. 59, 672 (1987); N. Masuhara, J.M. Doyle, J.C. Sandberg, D. Kleppner, T.J. Greytak, H.F. IIess, G.P. Kochanski, Phys. Rev. Lett. 61, 935 (1988).

[4] R. van Roijen, J.J. Berkhout, S. Jaakkola, J.T.M. Walraven, Phys. Rev. Lett. 61, 931 (1988).

[5] C. Wieman, E. Cornell, private communication.

[6] G. Lenz, P. Meystre, E. Wright, Phys. Rev. Lett. 71, 3271 (1993).

[7] H.D. Politzer, Phys. Rev. A 43, 6444 (1991).

[8] B. Svistunov, G. Shlyapnikov, Zh. Eksp. Teor. Fiz. 97, 821 (1990) [Sov. Phys. JETP 70, 460 (1990)]; ibid. 98, 129 (1990) [71, 71 1990].

[9] J. Javanainen, submitted to Phys. Rev. Lett., 1993.

[10] M. Lewenstein, L. You, Phys. Rev. Lelt. 71, 1339 (1993).

[11] L. You, M. Lewenstein, J. Cooper, submitted to Phys. Rev. Lelt., 1993.

[12] J.I. Cirac, M. Lewenstcin, P. Zoller, submitted to Phys. Rev. Lett., 1993.

[13] C.R. Monroe, E.A. Cornell, C.A. Sackett, C.J. Myatt, C. Wieman, Phys. Rev. Lett. 70, 414 (1993).

[14] S.R. de Groot, G.J. Hooyman, C.A. ten Seldam, Proc. R. Soc. Lond. A 203, 266 (1950).

[15] A. Einstein, Sitz.ber. Preuss. Acad. Wiss. Ber. 3, 18 (1925).

[16] G.E. Uhlenbeck, Over stalistische methoden in de theorie der quanta, Martinuus Nijhoff, The Hague 1927.

[17] F. London, Nalure 141, 643 (1938); Phys. Rev. 54, 947 (1938); J. Chem. Phys. 43, 49 (1939).

[18] V. Bagnato, D.E. Pritchard, D. Ḱleppner, Phys. Rev. A 35, 4354 (1987).

[19] G.D. Mahan, Many-Particle Physics, Plenum Press, New York 1993.

[20] N.N. Bogolyubov, J. Phys. Moscow 11, 231 (1947). 
[21] O. Penrose, Philos. Mag. 42, 1373 (1951); C.N. Yang, Rev. Mod. Phys. 34, 694 (1962).

[22] D. IIulin, A. Mysyrowicz, C. Benoît à la Guillaume, Phys. Rev. Lelt. 45, 1970 (1980).

[23] J.L. Lin, J.P. Wolfe, Phys. Rev. Lett. 71, 1222 (1993).

[24] J,M. Doyle, J.C. Sandberg, I.A. Yu, C.L. Cesar, D. Kleppner, T.J. Greytak, Phys. Rev. Lett. 67, 603 (1991); I.D. Setija, H.G.C. Werij, O. J. Luiten, M.W. Reynolds, T.W. Hijmans, J.T.M. Walraven, Phys. Rev. Lett. 70, 2257 (1993).

[25] J.J. Tollet, C.C. Bradley, R.G. Hulet, Bull. Am. Phys. Soc. 37, 1126 (1992).

[26] Private communications: E. Cornell, K. Burnett, C. Wieman, C.J. Myatt; see also, R.V.E. Lovelace, T.J. Tommila, Phys. Rev. A 35, 3597 (1987).

[27] M. Lewenstein, L. You, J. Cooper, R. Burnett, to be published.

[28] E.A. Power, Mathematical Physics Series, Vol. 24, Introductory Quantum Electrodynamics, Longmans, London 1964.

[29] E.A. Power, S. Zienau, Philos. Trans. R. Soc. 251, 427 (1959).

[30] J.R. Taylor, Scattering Theory: The Quantum Theory of Nonrelativistic Collisions, Wiley, New York 1987.

[31] R.H. Dicke, Phys. Rev. 89, 472 (1953); S.G. Rautian, I.I. Sobel'man, Sov. Phys. Usp. 9, 701 (1967).

[32] K. Rzążewski, M. Florjańczyk, J. Phys. B 17, L509 (1984); E.J. Robinson, P.R. Berman, J. Phys. B 17, L847 (1984).

[33] K. Rzążewski, Phys. Rev. A 28, 2565 (1983); M. Lewenstein, J. Zakrzewski, K. Rzążewski, J. Opt. Soc. Am. B 3, 22 (1986); M. Florjariczyk, K. Rzążewski, J. Zakrzewski, Phys. Rev. A 31, 1558 (1985); J. Zakrzewski, J. Phys. B 19, 224 (1986); E.J. Robinson, P.R. Berman, J. Phys. B 17, L847 (1984).

[34] C.W. Gardiner, Quantum Noise, Springer Verlag, Berlin 1991. 